UDHEP-10-92

IUHET-228

\title{
NEUTRINO MIXING DUE TO A VIOLATION OF THE EQUIVALENCE PRINCIPLE
}

\author{
J. Pantaleone \\ Physics Department \\ Indiana University, Bloomington, IN 47405 \\ and \\ A. Halprin, C. N. Leung \\ Department of Physics and Astronomy \\ University of Delaware, Newark, DE 19716
}

\begin{abstract}
Massless neutrinos will mix if their couplings to gravity are flavor dependent, i.e., violate the principle of equivalence. Because the gravitational interaction grows with neutrino energy, the solar neutrino problem and the recent atmospheric neutrino data may be simultaneously explained by violations at the level of $10^{-14}$ to $10^{-17}$ or smaller. This possibility is severely constrained by present accelerator neutrino experiments and will be preeminently tested in proposed long baseline accelerator neutrino experiments.
\end{abstract}


Several years ago, Gasperini noted that if the gravitational couplings of neutrinos are flavor dependent, mixing will take place when neutrinos propagate through a gravitational field [1]. Similar ideas were proposed independently by Halprin and Leung [2]. Consequently, experiments designed to search for neutrino mixing also probe the validity of the equivalence principle. In this Letter, we analyze the implications of present neutrino mixing experiments for the equivalence principle.

We consider the effects on neutrinos when they propagate under the influence of a weak, static gravitational field. For simplicity, we shall assume two neutrino flavors and neglect any neutrino masses. Ignoring effects which involve a spin flip, the flavor evolution of a relativistic neutrino is quite simple (see [2, 3] for more rigorous derivations). In the rest frame of a massive object, a neutrino has the effective interaction energy

$$
H=-2|\phi(r)| E(1+f)
$$

where $\mathrm{E}$ is the neutrino energy, and $\phi(r)=-|\phi(r)|$ is the Newtonian gravitational potential of the object. $f$ is a small, traceless, $2 \times 2$ matrix which parametrizes the possibility of gravity coupling to neutrinos with a strength different from the universal coupling, i.e. violations of the equivalence principle.

$f$ will be diagonal in some basis which we denote as the gravitational interaction basis (G-basis). In that basis, $\delta \equiv f_{22}-f_{11}$, then provides a measure of the degree of violation of the equivalence principle. In general, as occurs for neutrino masses, the flavor basis or the weak interaction basis (W-basis) will not coincide with the G-basis. If we denote the neutrino fields 
in the G-basis by $\nu_{G}=\left(\nu_{1}, \nu_{2}\right)$ and neutrinos in the $\mathrm{W}$-basis by $\nu_{W}=\left(\nu_{e}, \nu_{\mu}\right)$, $\nu_{G}$ and $\nu_{W}$ are related by a unitary transformation, $U^{\dagger}$ :

$$
\left(\begin{array}{c}
\nu_{1} \\
\nu_{2}
\end{array}\right)=\left[\begin{array}{cc}
\cos \Theta_{G} & -\sin \Theta_{G} \\
\sin \Theta_{G} & \cos \Theta_{G}
\end{array}\right]\left(\begin{array}{c}
\nu_{e} \\
\nu_{\mu}
\end{array}\right),
$$

where $\Theta_{G}$ is the mixing angle. Consequently when a massless neutrino propagates through a gravitational field, flavor mixing will occur.

The idea of using degenerate particles to study possible violations of the equivalence principle is not new. Similar effects have been considered in the neutral kaon system [4] for over 30 years. Note, however, that a violation of the equivalence principle in the kaon system requires that gravity couples differently to particles and antiparticles, a violation of CPT symmetry. This requirement is not necessary for neutrinos. Here, gravity is coupling slightly differently to different fermion generations.

Using Eq. (11), we may write down the flavor evolution equation for relativistic neutrinos propagating through a gravitational field (with no matter present). In the $\mathrm{W}$-basis, it reads

$$
i \frac{d}{d t}\left(\begin{array}{c}
\nu_{e} \\
\nu_{\mu}
\end{array}\right)=E|\phi(r)| \delta \quad U\left[\begin{array}{cc}
-1 & 0 \\
0 & 1
\end{array}\right] U^{\dagger}\left(\begin{array}{c}
\nu_{e} \\
\nu_{\mu}
\end{array}\right) .
$$

where we have neglected the irrelevant term in the hamiltonian which leads to an unobservable phase. For constant $\phi$, the survival probability for a $\nu_{e}$ after propagating a distance $\mathrm{L}$ is

$$
P\left(\nu_{e} \rightarrow \nu_{e}\right)=1-\sin ^{2}\left(2 \Theta_{G}\right) \sin ^{2}\left[\frac{\pi L}{\lambda}\right]
$$

where

$$
\lambda=6.2 \mathrm{~km}\left(\frac{10^{-20}}{|\phi| \delta}\right)\left(\frac{10 G e V}{E}\right)
$$


is the oscillation wavelength.

Eq. (4) is quite similar to that for vacuum oscillations due to neutrino masses (see e.g. [5]). However note the linear dependence of the oscillation phase on the neutrino energy. For a mass, the phase depends on $1 / E$. Thus these two sources of mixing can be easily distinguished by searching for neutrino mixing at different energies.

When the neutrino propagates through matter, then the mixing can be dramatically enhanced. A resonance occurs when

$$
\sqrt{2} G_{F} N_{e}=2 E|\phi| \delta \cos \left(2 \Theta_{G}\right)
$$

where $G_{F}$ is Fermi's constant and $N_{e}$ is the electron density. This effect is completely analogous to the well studied situation in which the mixing is due to neutrino masses ([6], for a review, see [5]). The survival probabilities for gravitationally induced mixing when there is a matter background can be obtained from those for masses by the transformation

$$
\frac{m_{2}^{2}-m_{1}^{2}}{4 E} \rightarrow E|\phi| \delta,
$$

if $\phi$ is a constant.

The local potential, $\phi$, enters through the phase of the oscillations, Eq. (4). It vanishes far from all sources of gravity so that results of special relativity are recovered. However the local value of $\phi$ is uncertain, because the estimates tend to increase as one looks at matter distributions at larger and larger scales. The potential at the Earth due to the Sun is $1 \times 10^{-8}$, that due to the Virgo cluster of galaxies is about $1 \times 10^{-6}$ while that due to our supercluster [4] has recently been estimated to be about $3 \times 10^{-5}$ (which 
is larger than $\phi$ in the Sun due to the Sun). In what follows, we will quote values for the combined, dimensionless parameter $|\phi| \delta$.

We now consider what the current data on neutrino mixing imply for the parameters $|\phi| \delta$ and $\Theta_{G}$. The searches for mixing can be divided into three broad categories; laboratory experiments, atmospheric neutrino observations and solar neutrino observations. The latter two have shown some evidence for neutrino mixing and will be considered first.

Solar neutrinos have been observed in four experiments [8], their results are summarized in Table 1. The observations are all well below the predictions of the standard solar model [9]-an indication of mixing. There are two mechanisms by which neutrino mixing can give large reductions in the solar neutrino flux, long-wavelength oscillations or resonant conversion. We shall consider these mechanisms separately.

If the distance between the Sun and the Earth is half of an oscillation wavelength and the mixing angle is large, then Eq. (4) predicts a large reduction in the flux. This occurs for $10 \mathrm{MeV}$ neutrinos when $|\phi| \delta=2 \times 10^{-25}$. Then the high energy neutrinos will be depleted but the lower energy neutrinos will be completely unaffected. However, the present data indicate mixing for the low energy solar neutrinos as well, see Table 1. A careful $\chi^{2}$ analysis finds that there is no long-wavelength, two flavor explanation of the data-it is disfavored at the 3 standard deviation level. This is in contrast to the normal case of mixings induced by mass differences for which the data are well described by vacuum oscillations of two flavors (see e.g. [10]). The difference is due to the differing energy dependence of the two types of mixings. Next generation solar neutrino observations [1] will further constrain 
this possibility by measuring the solar neutrino energy dependence (SNO or Super-Kamiokande) and by searching for seasonal variations (BOREXINO).

Resonant conversion as the neutrinos propagate through the interior matter of the Sun can also lead to large reductions in the flux. Figure 1 shows the favored regions from a $\chi^{2}$ fit to the flux reduction values in Table 1 (the Kamiokande-II energy bins are included explicitly and their overall systematic error is correctly accounted for [8]). An analytical expression was used to describe the resonant conversion survival probability [5, 3], based on only the Sun's gravitational potential. The average gravitational potential in the sun, $|\bar{\phi}|=4 \times 10^{-6}$, was used to normalize Fig. (1). If a constant potential from larger scales dominates over this, then the allowed regions in Figure 1 are slightly elongated about the mean $|\bar{\phi}| \delta$ by approximately a factor of 3 . Next generation solar neutrino observations will test these regions [3] by measuring the solar neutrino energy dependence (SNO or Super-Kamiokande), by looking for day-night variations, and by performing a neutral current measurement of the solar flux (SNO).

Two experiments [7] recently found that there is a relative depletion of $\nu_{\mu}$ to $\nu_{e}$ in the flux of low energy atmospheric neutrinos. This depletion may be the result of flavor mixings. The energy of the atmospheric neutrinos is typically about $0.5 \mathrm{GeV}$. The propagation length now varies from 20 to 10,000 kilometers. This corresponds to values of $|\phi| \delta$ from about $6 \times 10^{-20}$ to about $10^{-22}$. Although the data can be explained by either $\nu_{\mu}-\nu_{e}$ mixing or $\nu_{\mu}-\nu_{\tau}$ mixing, and so do not necessarily probe the same parameters as solar neutrinos, it is encouraging that a range of $|\phi| \delta$ can account for the solar neutrino data and the atmospheric neutrino data simultaneously [3]. 
This may signal a possible breakdown of the principle of equivalence.

Numerous laboratory experiments have been performed searching for neutrino mixing. They have not found definitive evidence for mixing, so these experiments eliminate ranges of the gravitationally induced mixing parameters. The most stringent laboratory limits come from "appearance" experiments with the largest values of $E \times L$. These occur in experiments using the beams of neutrinos produced by accelerators $[12$, where the neutrino energies are tens of $\mathrm{GeV}$ and propagation lengths are as long as a kilometer. Because most mixing experiments only analyze the data in terms of $\frac{L}{E}$, the relevant quantity for neutrino masses, the results are not exactly transferable to gravitationally induced mixing. From the published descriptions of the experiments, we have estimated the average value of $E \times L$ to derive the limits shown in the top part of Fig. (2). Our estimates are probably accurate up to factors of 3 in $|\phi| \delta$.

Most of the large mixing angle region which solves the solar neutrino problem is eliminated by the current laboratory bounds. But the lower part of this solution, and the small mixing angle region, are still allowed by the present accelerator data. Only relatively small improvements in the current bounds are needed to constrain these regions.

New accelerator neutrino experiments, with baselines of hundreds or thousands of kilometers, are under active consideration at the present time. In the lower part of Fig. (1) are shown estimates of the accessible parameter region achievable by two of the proposed experiments [13], FNAL to Soudan 2 and FNAL to DUMAND. At these long distances matter effects are becoming important [14], as is apparent in the difference between $\nu_{\mu}$ and $\bar{\nu}_{\mu}$ 
regions. The energy distribution of the neutrino event rate is taken from calculations for a short baseline experiment using the FNAL main injector. Also, similar neutrino energies, intensities and propagation lengths are available to planned next generation atmospheric neutrino detectors (such as DUMAND, AMANDA, etc.). They may be able to probe parameter regions similar to those shown for the accelerator experiments. Thus there are many planned and proposed experiments which can extend the tests of the equivalence principle to values of $|\phi| \delta$ far below the present accelerator limits.

In conclusion, the degeneracy of neutrinos enables tests of the equivalence principle which are far more sensitive than those using "normal" matter [15]. The present solar neutrino data suggest a violation of the equivalence principle at the level of $2 \times 10^{-19}<|\phi| \delta<3 \times 10^{-22}$. The atmospheric neutrino data also suggest a possible breakdown of the equivalence principle at this same level. This possibility can be independently checked by longbaseline accelerator neutrino experiments, which can reach down to $|\phi| \delta \approx$ $10^{-24}$. The violation of the equivalence principle is introduced on purely phenomenological grounds. Such a violation would indicate a breakdown in general relativity or the existence of additional long range tensor interactions.

We thank M. Butler, S. Nozawa, R. Malaney and A. Boothroyd for communicating preliminary results from their analysis. CNL wishes to thank Fermilab for their hospitality and the University of Delaware Research Foundation for partial support. JP thanks Brookhaven and the Queen's University Summer Institute for their hospitality. This work was supported in part by the U.S. Department of Energy under Grants No. DE-FG02-84ER40163 and DE-FG02-91ER40661. 


\section{References}

[1] M. Gasperini, Phys. Rev. D 38, 2635 (1988); Phys. Rev. D 39, 3606 (1989).

[2] A. Halprin and C. N. Leung, Phys. Rev. Lett. 67, 1833 (1991); Nucl. Phys. B (Proc. Suppl.) 28A, 139 (1992).

[3] A. Halprin, C. N. Leung, and J. Pantaleone, in preparation.

[4] R. J. Hughes, Phys. Rev. D 46, R2283 (1992); I. R. Kenyon, Phys. Lett. B 237, 274 (1990); M.L. Good, Phys. Rev. 121, 311 (1961).

[5] T.K. Kuo and J. Pantaleone, Rev. Mod. Phys. 61, 937 (1989).

[6] L. Wolfenstein, Phys. Rev. D 17, 2369 (1978); Phys. Rev. D 20, 2634 (1979). S. P. Mikheyev and A. Yu Smirnov, Yad. Fiz. 42, 1441 (1985) [Sov. J. Nucl. Phys. 42, 913 (1985)]; Nuovo Cim. C9, 17 (1986).

[7] K. S. Hirata et al., Phys. Lett. B 280, 146 (1992). D. Casper et al., Phys. Rev. Lett. 66, 2561 (1991). E. W. Beier et al., Phys. Lett. B 283, 446 (1992).

[8] R. Davis, D.S. Harmer and K.C. Hoffman, Phys. Rev. Lett. 20, 1205 (1968); R. Davis, in Proc. of the 21st Int. Cosmic Ray Conf., ed. by R.J. Protheroe (University of Adelaide Press) 143 (1990). K. Hirata et al., Phys. Rev. Lett. 65, 1297 (1990); 1301 (1990); Phys. Rev. D44, 2241 (1991). A.I. Abazov et al. (SAGE), Phys. Rev. Lett. 67, 3332 (1991). 
A. Gavrin, talk presented at Dallas Conference. P. Anselmann et al. (GALLEX), Phys. Lett. B285, 376 (1992); 390 (1992).

[9] J.N. Bahcall and R.K. Ulrich, Rev. Mod. Phys. 60, 297 (1988); J.N. Bahcall and M.H. Pinsonneault, Rev. Mod. Phys., Oct. (1992).

[10] A. Acker, S. Pakvasa, and J. Pantaleone, Phys. Rev. D 43, R1754 (1991). V. Barger, R.J.N. Phillips and K. Whisnant, University of Wisconsin preprint $\mathrm{MAD} / \mathrm{PH} / 708$.

[11] H.H. Chen, Phys. Rev. Lett. 55, 1534 (1985). G.T. Ewan et al., Sudbury Neutrino Observatory (SNO) Proposal, October (1987). Y. Totsuka (Super-Kamiokande), Tokyo Univ. preprint ICRR-227-90-20. C. Arpesella, et al., BOREXINO at Gran Sasso, August (1991).

[12] N.J. Baker et al., Phys. Rev. Lett. 47, 1577 (1981). N. Ushida et al., Phys. Rev. Lett. 57, 2897 (1986); Ahrens et al., Phys. Rev. D31, 2732 (1985).

[13] V.J. Stenger (DUMAND), Proceedings of the Workshop on Long Baseline Neutrino Oscillations, Fermilab (1991) p. 317; E.A. Peterson (Soudan 2) ibid. p. 243.

[14] J. Pantaleone, Phys. Lett. B292, 201 (1992); B246, 245 (1990); R.H. Bernstein and S.J. Parke, Phys. Rev. D44, 2069 (1991).

[15] V.B. Braginsky and V.I. Panov, Zh. Eksp. Teor. Fiz. 61, 873 (1971) [Sov. Phys. JETP, 34, 463 (1972)]. More recent experiments have not improved this limit, see, e.g., B.R. Heckel et al., Phys. Rev. Lett. 63, 
2705 (1989). M.J. Longo, Phys. Rev. Lett. 60, 173 (1988). S. Pakvasa, W.A. Simmons and T.J. Weiler, Phys. Rev. D39, 1761 (1989). 
Table 1. Results of the solar neutrino experiments [8]. The flux is given as a fraction of the standard solar model [9] prediction.

\begin{tabular}{|l|l|l|l|}
\hline Experiment & Process & $\mathrm{E}_{\text {threshold }}$ & Expt./SSM \\
\hline Davis et al. & $\nu_{e}+{ }^{37} \mathrm{Cl} \rightarrow e+{ }^{37} \mathrm{Ar}$ & $0.81 \mathrm{MeV}$ & $0.27 \pm 0.04$ \\
Kamiokande-II & $\nu+e \rightarrow \nu+e$ & $7.5 \mathrm{MeV}$ & $0.46 \pm 0.05 \pm 0.06$ \\
SAGE & $\nu_{e}+{ }^{71} \mathrm{Ga} \rightarrow e+{ }^{71} \mathrm{Ge}$ & $0.24 \mathrm{MeV}$ & $0.44{ }_{-0.18}^{+0.13} \pm 0.11$ \\
GALLEX & $\nu_{e}+{ }^{71} \mathrm{Ga} \rightarrow e+{ }^{71} \mathrm{Ge}$ & $0.24 \mathrm{MeV}$ & $0.63 \pm 0.14 \pm 0.06$ \\
\hline
\end{tabular}




\section{FIGURE CAPTION}

Fig. 1. $\chi^{2}$ plot showing regions of $|\bar{\phi}| \delta$ versus $\sin ^{2} 2 \Theta_{G}$ allowed by the solar neutrino data in Table 1 at $90 \%$ (solid lines) and 99\% (dotted lines) confidence level, assuming two flavors and $\delta>0$.

Fig. 2. Upper contours: Regions of $|\phi| \delta$ versus $\sin ^{2} 2 \Theta_{G}$ that are excluded by accelerator neutrino data [12]. Lower contours: Regions probed by proposed [13] long-baseline experiments, assuming sensitivity to $10 \% \nu_{\mu}\left(\overline{\nu_{\mu}}\right)$ disappearance. The outer dashed (inner dash dot) curve is for a $\nu_{\mu}\left(\bar{\nu}_{\mu}\right)$ beam from FNAL $\rightarrow$ DUMAND. The inner dashed (dot) curve is for a $\nu_{\mu}$ $\left(\bar{\nu}_{\mu}\right)$ beam from FNAL $\rightarrow$ Soudan 2. 